\title{
Study on Deformation and Failure Characteristics of Weak Cemented Roof in Shallow and Extra Thick Coal Seam Mining
}

\section{Chang Liu}

Anhui University of Science and Technology https://orcid.org/0000-0001-8679-1234

Pingsong Zhang ( $\sim$ pszhang@aust.edu.cn)

Anhui University of Science and Technology

Duoxi Yao

Anhui University of Science and Technology

Yuancaho Ou

Anhui University of Science and Technology

Yutong Tian

Anhui University of Science and Technology

\section{Research Article}

Keywords: Weak cemented roof, Hydraulic fracture, Dual-mode parallel electrical method, Apparent resistivity, Ground fissure

Posted Date: August 4th, 2021

DOl: https://doi.org/10.21203/rs.3.rs-713921/v1

License: (c) (1) This work is licensed under a Creative Commons Attribution 4.0 International License. Read Full License 


\title{
Study on deformation and failure characteristics of weak cemented roof in shallow and extra thick coal seam mining
}

\author{
Chang Liu ${ }^{\mathrm{a}, \mathrm{b}}$, Pingsong Zhang ${ }^{\mathrm{a}, \mathrm{b}, *}$, Duoxi Yao ${ }^{\mathrm{a}, \mathrm{b}}$, Yuanchao Ou ${ }^{\mathrm{a}, \mathrm{b}}$ and Yutong Tian ${ }^{\mathrm{a}, \mathrm{b}}$ \\ ${ }^{\text {a }}$ State Key Laboratory of Mining Response and Disaster Prevention and Control in Deep Coal Mines , Anhui \\ University of Science and Technology, Huainan City, Anhui Province, 232001, China \\ ${ }^{\mathrm{b}}$ School of Earth and Environment, Anhui University of Science and Technology, Huainan City, Anhui Province, \\ 232001, China
}

*Corresponding author: Pingsong Zhang, E-mail: pszhang@aust.edu.cn

\begin{abstract}
Detecting the development height of water flowing fractured zone in the roof of coal seam is of great significance for the roof safety of working face with developed sandstone and thick loose layer. This paper analyzes the influence of the induced polarization effect of electrode on the traditional single-mode parallel electrical method. In order to avoid this interference, the dual-mode parallel electrical method is applied to monitor the roof deformation and failure of a coal mine in Ordos Basin. The monitoring results show that: under the influence of mining, the change of geoelectric field of coal seam roof is dynamic, the apparent resistivity of initial mining fracture is high, the apparent resistivity of surrounding rock water filling mining fracture is low, and the apparent resistivity of fracture water flowing to goaf is high again. According to the characteristics of geoelectric field, the maximum height of water flowing fracture zone is $122 \mathrm{M}$; The average mining coal thickness of the working face is $11 \mathrm{~m}$, and the fracture / mining thickness ratio is 11.1. The results of plastic zone show that the maximum height of water flowing fractured zone above the working face is $122 \mathrm{~m}$, which is consistent with the test results. The vertical stress response characteristics of roof monitoring points are zonal. Under the influence of mining, the loose layer near the surface above the working face is cracked. The edge type ground fissures exist for a long time, and there are a series of geological problems such as soil erosion, ground subsidence.
\end{abstract}

Keywords Weak cemented roof . Hydraulic fracture - Dual-mode parallel electrical method . Apparent resistivity . Ground fissure

\section{Introduction}

Western China is an important exporter of coal resources and the geological conditions of its coal mining areas are mainly characterized by thick Quaternary loose beds, deep buried soft bedrock, and rich aquifers (Fu Y P et al. 2012). Mining causes stress redistribution of the surrounding rock of the working face; and the deformation and failure of the rock and soil mass at the upper part of the working face caused by this is a nonlinear and multi-factor-controlled geological disaster phenomenon (Chen et al. 2018; Yu et al. 2019). This can lead to surface soil erosion, landslides, ground subsidence, cracks, underground cavities, and other geological problems.

Scholars at home and abroad have done a lot of research on roof deformation and failure under mining conditions, and achieved fruitful results. Coal seam roof research can be broadly divided into four domains: theoretical analyses, numerical simulations, physical similarity modeling, and 
field measurements. In terms of theoretical analyses, the "Three-Zone" theory has been widely applied in the deformation and failure analysis of coal face roofs. According to the deformation and failure strength and range of overburden (Qian et al. 2003), it can be divided into caving zone, fracture zone, and bending subsidence zone from bottom to top. For the roof with a thick loose layer and thin overburden, it can be further summarized into two stages: the deformation, fracture, and displacement of the lower strata and the deformation and displacement of the upper strata (Wang et al. 2000). Li et al. (2003) calculated the movement and deformation distribution of overburden strata in shallow buried coal seam mining by the probability integral method. Xu et al. (2005) proposed a method to predict the failure height of overburden based on the position of key strata. Guo et al. (2019) put forward a new method to predict the height of the fracture water conduction zone in high strength longwall coal mining. Huang et al. (2017) proposed a new method for predicting the height of the water-conducting fractured zone based on composite structure overburden and tensile deformation calculation of rock strata. In terms of numerical simulation, An et al. (2010) adopted the RFPA numerical simulation method to analyze the movement law of overlying rock and the development of the water-conducting fracture zone in the 31401 fully-mechanized mining face of Bulianta Coal Mine in Shendong Mine Area. The results showed that the composite water-resisting key layer formed in the mining process can effectively protect the aquifer and prevent the loss of water resources. Liu et al. (2016) used UDEC numerical simulation software to analyze the dynamic development rules of sliding ground fractures caused by mining, and proposed the concept of sliding type fractures and fracture angles. In terms of physical similarity model experiments, Chai Jing et al. (2021) combined BOTDA, FBG, and DIC optical measurement methods to jointly study the deformation and failure law of overburden rock in coal mine stope through different testing methods of "point-line-plane". Zhang et al. (2017) monitored and analyzed the development rules of overburden deformation and failure in the process of coal mining, and obtained the corresponding relationship between strain distribution and stratum; the strain change of the sensing optical cable was inversely proportional to the modulus of overburden. Fracture evolution laws, mining-induced deformation, and stress distribution characteristics of composite KAS with different BMCRs are studied (Sun et al. 2013). In terms of field measurements, these can be divided into direct observation methods and indirect observation methods. Direct observation methods include the drilling TV method and the drilling flushing fluid method (Sun et al. 2013). Indirect observation methods include electrical monitoring technology (Wu et al. 2020; Ou et al. 2020), the acoustic emission monitoring method (AE) (Liu et al. 2020), the microseismic monitoring system (MS) (Xia et al. 2021), and optical fiber testing technology (Zhou et al. 2019). In-situ testing technology is gradually developing in a real-time and accurate direction. There are some applications of the parallel electric method to detect the stability of overlying rock in stope, but almost all of them use a single electrode as power supply and collection. The accuracy of this method will be negatively affected by the phenomenon of excited polarization, which is analyzed in detail in Section "Dual-mode parallel electric monitoring technology". The super long surface electrical survey line is not common.

\section{Overview of working face and technology principle}

\section{Overview of working face}

The working face of the coal mine in Ordos Basin is located in the middle of the east wing area of 
the mine field. The west of the working face is the deputy inclined shaft and the NO. 5 coal belt machine lane. The south is the unexcavated area; $30 \mathrm{~m}$ in the north is the 509 working face under tunneling construction. The east is near the boundary of the mine field. The surface of this working face is the Loess Plateau with an elevation of $+1173.0 \sim+1322.0 \mathrm{~m}$. It is mainly composed of loess beams, hills, and gullies, with the development of dendritic gullies. The terrain is undulating and there is no water body on the surface and no outcropping of bedrock. The No. 5 coal seam is located in the lower member of Shanxi Formation of the lower Permian system. According to comprehensive data from nearby boreholes, the average coal thickness of 5 coal seam is $11.0 \mathrm{~m}$, the strike length of the working face is $2885 \mathrm{~m}$, the dip width is $250 \mathrm{~m}$, and the dip angle of the coal seam is generally $3^{\circ} \sim 7^{\circ}$. The old roof of the No.5 coal seam is a mud-cemented and loose coarse sandstone aquifer with an average thickness of $7.1 \mathrm{~m}$, and the local phase is transformed into gravel, coarse sandstone, or conglomerate, which is the direct and main water-filled aquifer in the process of mining the No. 5 coal seam. Fig. 1 shows the borehole core columnar and well logging curves.

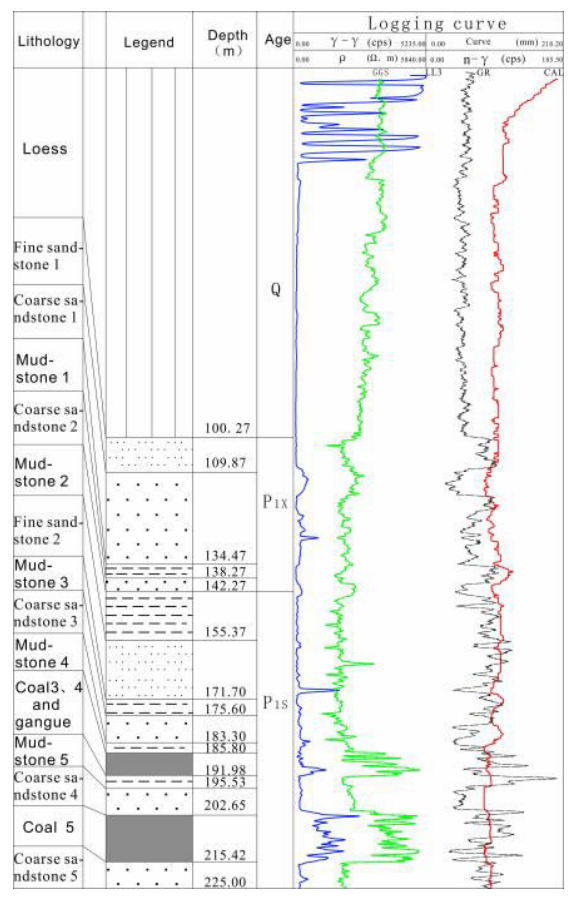

Fig 1: Column chart and logging curve of overburden borehole in madiliang 507 working face

\section{Dual-mode parallel electric monitoring technology}

The mine parallel electrical method is a new generation of electrical data acquisition technology developed after high-density electrical method. The parallel electric method can obtain data from multiple measuring points simultaneously for each power supply. It is a full electric field observation technology, which can collect spontaneous potential, primary field, and secondary field electrical data at the same time. By combining parallel acquisition technology, a network communication system, and an intelligent control system, it can be applied to the deformation and failure monitoring of overburden and the real-time analysis of the resistivity change in three-dimensional space. The monitoring system in this paper selects WBD-2 parallel electrical method instrument for the mine network, and the data acquisition method is the "AM" method. In the "AM" method, the public ground electrode $\mathrm{N}$ and the negative electrode $\mathrm{B}$ of the public power 
supply are separately laid. $\mathrm{N}$ electrodes on the survey line automatically take turns as the positive electrode A of the power supply, and the remaining n-1 electrodes automatically play the role of the collection electrode M (Liu et al. 2019).

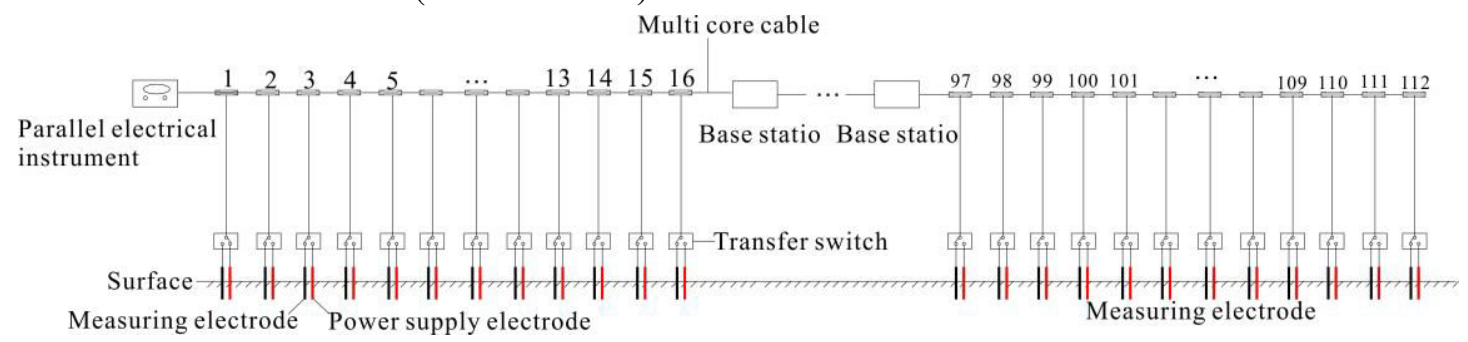

Fig.2 Schematic diagram of electrode control for dual-mode parallel electrical method

Different from the single-mode parallel electrical method with one electrode at each measuring point, the dual-mode parallel electrical method has two electrodes at each measuring point. When data are collected at each measuring point, the two electrodes are single power supply and single collection (Fig. 2) to minimize the influence of the electrode-induced polarization effect and ensure stable and accurate data collection (Yang et al. 2019). In this paper, the advantages of the dual-mode parallel electrical testing technique over the single-mode parallel electrical testing technique are deduced theoretically.

According to Chen et al. (Chen et al. 2016) when the measured ground is a uniform non-polarized medium, the formula for calculating the apparent resistivity is as follows:

$$
\rho_{s}=K \frac{\Delta U_{1}}{I}
$$

In the formula, $\rho_{S}$ is the apparent resistivity for measurement, $K$ is the device coefficient, $\Delta U_{1}$ is the voltage between the electrodes for measurement, and $I$ is the supply current.

In the case of an underground polarized medium such as metal orebody, when the underground current flows through the orebody, a polarization reaction will occur in the orebody under the action of current, resulting in "secondary current" and an electric field around the orebody (Fig. 3). "Secondary potential difference" $\Delta U_{2}$ will be generated between the measuring electrode $\mathrm{M}$ and the measuring electrode $N$.
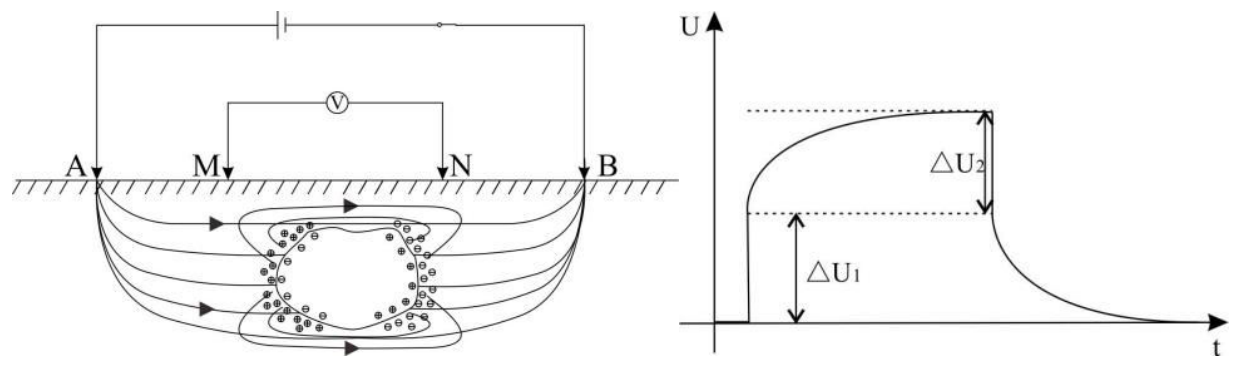

(a) Induced polarization phenomenon

(b) Charging and discharging curves of induced polarization in time domain

Fig 3: Components of network parallel electrical method test

$$
\rho_{S}^{\prime}=K \frac{\Delta U_{1}+\Delta U_{2}}{I}
$$

When $\mathrm{MN} \ll \mathrm{AB}$, the electric field intensity measured between the electrodes is considered to be uniform.

$$
\Delta U_{1}+\Delta U_{2}=\left(E_{1}+E_{2}\right) \cdot \overline{M N}
$$

In the formula, $E_{1}$ is the electric field intensity between electrode $M$ and electrode $N ; E_{2}$ is the 
additional electric field intensity caused by the induced polarization effect. $\overline{M N}$ is the distance between measuring electrode $M$ and measuring electrode $N$.

$$
E_{1}+E_{2}=\left(j_{1}+j_{2}\right) \cdot \rho_{M N}
$$

In the formula, $j_{1}$ is the current density between electrode $M$ and electrode $N ; j_{2}$ is the current density of the additional electric field; $\rho_{M N}$ is the true resistivity of the medium in which $M N$ is located. Eqs (1)-(4) can be obtained as follows:

$$
\rho_{s}^{\prime}=K \frac{\left(j_{1}+j_{2}\right) \cdot \rho_{M N} \cdot \overline{M N}}{I}
$$

According to Eq. (4), the relationship between the apparent resistivity $\rho_{s}$ and $\rho_{M N}$ obtained by the test can be seen. The ground is regarded as a unit of countless uniform medium, and the resistivity of each unit is equal to the apparent resistivity, $\rho_{0}$, and the current density is $j_{0}$. The distribution of the unit body does not consider the effect of additional electric field, that is, $j_{1}=0$. Put it into Eq. (5) to get:

$$
\rho_{0}=K \frac{j_{0} \cdot \rho_{0} \cdot \overline{M N}}{I}
$$

Eq. (6) can be obtained by sorting out.

$$
\frac{1}{j_{0}}=K \frac{\overline{M N}}{I}
$$

Substitute Eq. (7) into Eq. (5) to get:

$$
\rho_{s^{\prime}}=\frac{\left(j_{1}+j_{2}\right) \cdot \rho_{M N}}{j_{0}}=\frac{j_{1} \cdot \rho_{M N}}{j_{0}}+\frac{j_{2} \cdot \rho_{M N}}{j_{0}}
$$

In the formula, $\rho_{S}$ ' is the measured apparent resistivity considering the induced polarization effect. If $\rho_{1}=\frac{j_{2} \cdot \rho_{M N}}{j_{0}}, \rho_{1}$ is the effect of electrode-induced polarization on the apparent resistivity measurement results. Based on this, it is known that the induced polarization caused by a single electrode leads to the deviation of the measurement results. The dual-mode parallel electric method can avoid this error and make the measurement results more accurate.

\section{Numerical Simulation}

To obtain the development of working face fractures in the process of coal mining, FLAC ${ }^{3 D}$ numerical simulation software is used to build a three-dimensional numerical model (Fig. 4a). The size of $800 \mathrm{~m} \times 400 \mathrm{~m} \times 226 \mathrm{~m}(\mathrm{x} \times \mathrm{y} \times \mathrm{z})$ based on the geological conditions of 507 working face, and the Mohr-Coulomb criterion is applied. There are fixed constraints around and at the bottom of the model. The thickness of each layer is shown in Fig. 1 "Initial" command is adopted for the initial horizontal stress with an initial value of -2.0 MPa and a gradient value of $0.015 \mathrm{MPa}$. According to the hydrogeological data, a certain water pressure is set in the roof sandstone, and monitoring points are set above the coal seams with $40 \mathrm{~m}$ and $248 \mathrm{~m}$ reared feet. The vertical spacing between adjacent monitoring points is $20 \mathrm{~m}$ (Fig. 4b). 


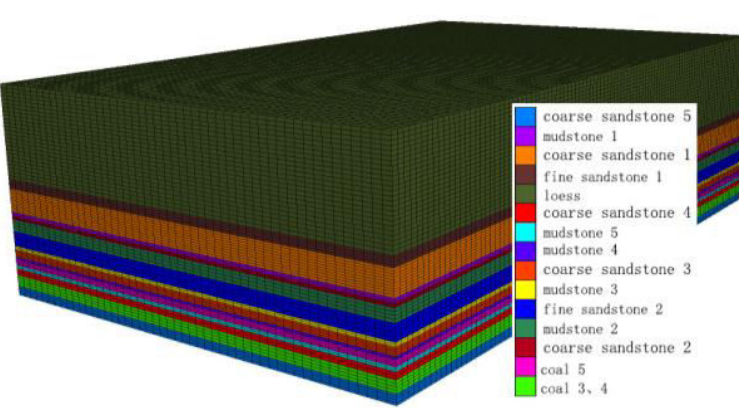

(a) 3D model

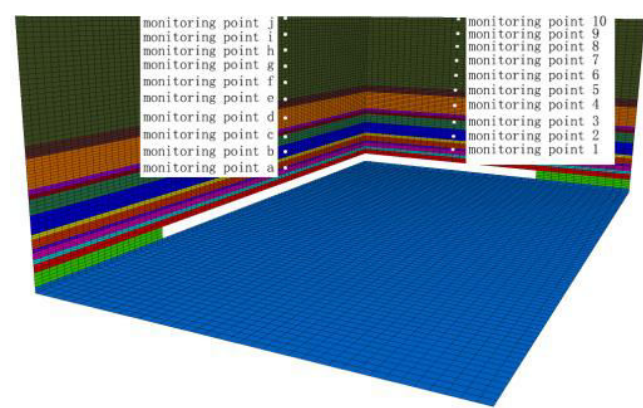

(b) Distribution of monitoring points

Fig 4: 3D numerical model and monitoring point setting

Through rock mechanics experiments, the physical and rock mechanics parameters of the roof and floor of a 507 working face in Ordos Basin were obtained, as shown in Table 1.

The entire height of the simulated excavation is mined at one time. The excavation step is $8 \mathrm{~m}$, and there are 62 excavation steps in total. The length of the working face's strike direction is 250 m. Fig. 5 shows the three-dimensional section of the plastic zone advancing at $256 \mathrm{~m}$ and $496 \mathrm{~m}$ of the working face. The results show that tensile failure mainly occurs in the upper and middle part of the goaf, while shear failure mainly occurs in the middle part. The shear stress failure zone appears in front of the coal wall of the working face. When the working face advances $256 \mathrm{~m}$, the maximum height of plastic zone of overburden is $106 \mathrm{~m}$. When the working face advances $496 \mathrm{~m}$, the maximum height of plastic zone of overburden is $122 \mathrm{~m}$. The upper limit of the overburden plastic zone can be regarded as the maximum height of the development of the water-conducting fracture zone. The analysis shows that the maximum water-conducting fracture of overburden is $122 \mathrm{~m}$.
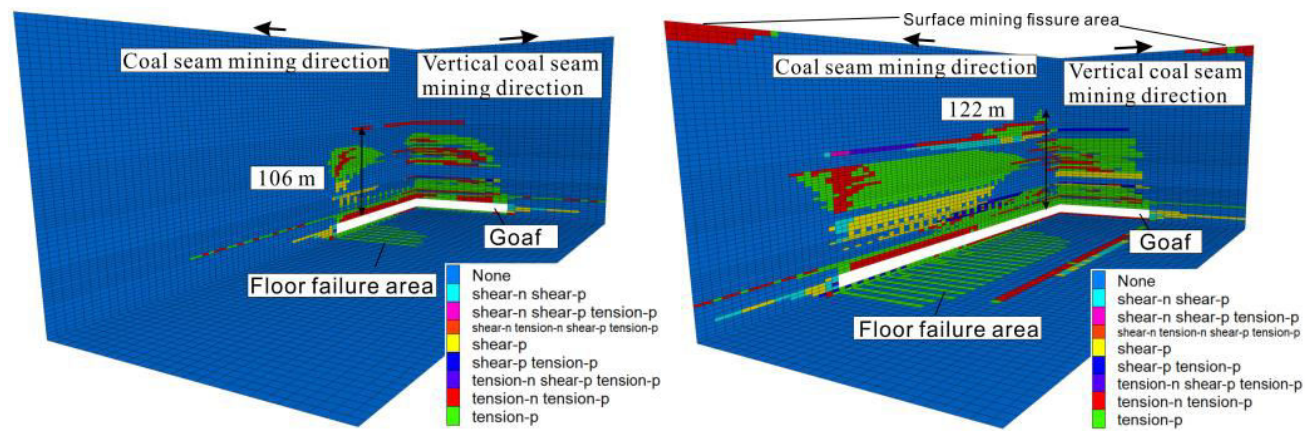

(a) Distribution of plastic zone in $256 \mathrm{~m}$ mining face

(b) Distribution of plastic zone in 496m mining face

Fig 5: Three-dimensional slicing of mining plastic zone in working face

The stress values in the vertical direction of each monitoring point in the process of mining are counted (Fig. 6). When no mining is carried out, the vertical stress values of each monitoring point are positively correlated with the buried depth. With the progress of mining, the vertical stress of monitoring points 1 and 2 above the goaf changes substantially, and they are "Active monitoring points". The vertical stress of monitoring points 3 - 6 only changes slightly, and they are "General active monitoring points". The vertical stress of monitoring points $7-10$ exhibits no obvious change, and they are "Stable monitoring points". Monitoring point 6 corresponds to the height of the roof at $120 \mathrm{~m}$. The rock and soil layer of the roof within the range of the height of the roof is greatly affected by mining, which provides conditions for the formation and development of rock caving and cracks. As the working face moves away from the goaf, the vertical stress of the 
monitoring point above the goaf gradually tends to become stable. The dynamics of the vertical stress of monitoring points A - F above the coal seam working face are as follows: The compressive stress increases - The tensile strain significantly increases - and Tends to be stable. Monitoring point $\mathrm{F}$ corresponds to the height of the coal seam roof of $120 \mathrm{~m}$. The vertical stress change process of monitoring points $\mathrm{F}$ and $\mathrm{H}$ consists of two stages: tensile stress increases and tends to be stable. The vertical stress of monitoring point $\mathrm{I}-\mathrm{J}$ exhibits no obvious change and is basically not affected by mining. The influence of mining on the vertical stress of monitoring points at different heights is different, and the distribution of vertical stress is zonal. 
Table 1: 507 physical and rock mechanical parameters of roof and floor strata of working face

\begin{tabular}{|c|c|c|c|c|c|c|c|c|}
\hline \multirow[b]{2}{*}{ Lithology } & \multicolumn{8}{|c|}{ Rock mechanical parameter } \\
\hline & $\begin{array}{l}\text { Elastic modulus / } \\
\text { (Gpa) }\end{array}$ & $\begin{array}{l}\text { Shear modulus / } \\
(\mathrm{GPa})\end{array}$ & $\begin{array}{c}\text { Cohesion / } \\
\text { (Mpa) }\end{array}$ & $\begin{array}{l}\text { Fric / } \\
\left({ }^{\circ}\right)\end{array}$ & $\begin{array}{c}\text { Tensile strength / } \\
(\mathrm{MPa})\end{array}$ & $\begin{array}{l}\text { Density / } \\
\left(\mathrm{Kg} / \mathrm{m}^{3}\right)\end{array}$ & $\begin{array}{l}\text { Permeability coefficient / } \\
\qquad(\mathrm{m} / \mathrm{s})\end{array}$ & Porosity \\
\hline Loess & 0.8 & 0.8 & 1.6 & 29.2 & 1.20 & 1580 & $5.8 \times 10^{-6}$ & 0.20 \\
\hline Fine sandstone 1 & 5.2 & 2.5 & 13.2 & 28.6 & 2.80 & 2658 & $2.2 \times 10^{-11}$ & 0.06 \\
\hline Coarse sandstone 1 & 4.2 & 2.4 & 6.5 & 31.2 & 1.66 & 2606 & $2.4 \times 10^{-11}$ & 0.10 \\
\hline Mudstone 1 & 5.8 & 2.3 & 10.0 & 31.6 & 1.6 & 2575 & $3.8 \times 10^{-12}$ & 0.04 \\
\hline Coarse sandstone 2 & 4.2 & 2.4 & 6.50 & 31.2 & 1.66 & 2606 & $2.4 \times 10^{-11}$ & 0.08 \\
\hline Mudstone 2 & 5.8 & 2.3 & 10.0 & 31.6 & 1.60 & 2575 & $3.0 \times 10^{-12}$ & 0.04 \\
\hline Fine sandstone 2 & 5.3 & 2.6 & 13.4 & 28.5 & 2.80 & 2662 & $2.0 \times 10^{-11}$ & 0.06 \\
\hline Mudstone 3 & 5.8 & 2.3 & 10.0 & 31.6 & 1.60 & 2575 & $3.3 \times 10^{-12}$ & 0.04 \\
\hline Coarse sandstone 3 & 4.4 & 2.6 & 6.8 & 31.2 & 1.76 & 2626 & $2.4 \times 10^{-11}$ & 0.08 \\
\hline Mudstone 4 & 5.8 & 2.3 & 10.0 & 31.6 & 1.60 & 2575 & $3.2 \times 10^{-12}$ & 0.04 \\
\hline NO. 3、 4 coal & 2.2 & 0.6 & 2.7 & 32.0 & 1.20 & 1575 & $8.0 \times 10^{-11}$ & 0.05 \\
\hline Mudstone 5 & 5.8 & 2.3 & 10.0 & 31.6 & 1.60 & 2575 & $3.0 \times 10^{-12}$ & 0.04 \\
\hline Coarse sandstone 4 & 4.5 & 2.7 & 6.8 & 30.8 & 4.80 & 2628 & $2.4 \times 10^{-11}$ & 0.10 \\
\hline NO. 5 coal & 2.2 & 0.6 & 2.7 & 32.0 & 1.20 & 1575 & $8.0 \times 10^{-11}$ & 0.05 \\
\hline Coarse sandstone 5 & 5.2 & 3.2 & 7.2 & 31.2 & 5.00 & 2714 & $1.0 \times 10^{-11}$ & 0.10 \\
\hline
\end{tabular}



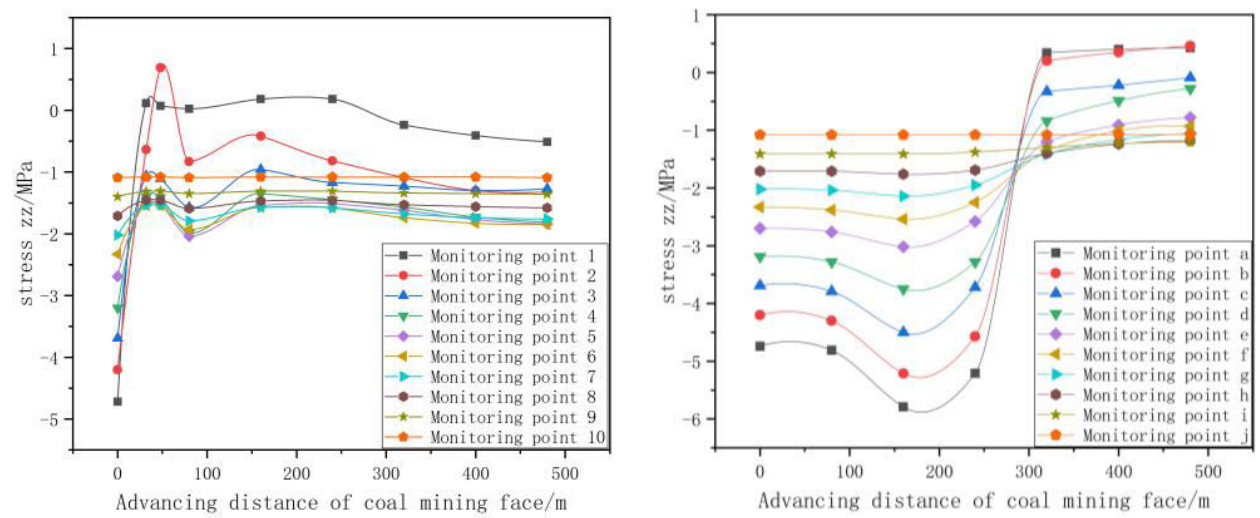

(a) Vertical stress curve of monitoring point above goaf

(b) Vertical stress curve of monitoring point on coal seam inclination direction

Fig 6: Vertical stress curve of model monitoring points

\section{In Situ Testing}

In the process of working face mining, the monitoring area of the survey line will be affected by the structural stress and the complex hydrogeological conditions of the roof. The electrical parameters of rocks in the monitoring range will change accordingly. The electrical observation system captures the changes in apparent resistivity and then monitors the dynamic changes of the overlying strata in real time.

To meet the task of detecting the law of roof fracture in the working face, two vertical crossover survey lines were designed and constructed on the surface above working face 507 (Fig. 7). Measurement line 1 was parallel to the direction of the mining face and included a total of 112 electrodes. The No. 1 electrode was at the incision and the No. 112 electrode was at the drawback of $504 \mathrm{~m}$. The vertical depth was controlled at $277.2 \mathrm{~m}$ (depth coefficient 0.55 ). Line 2 is perpendicular to the mining direction of the working face, with 112 electrodes. No. 1 and No. 2 electrodes were located at $40.4 \mathrm{~m}$ and the vertical depth was controlled at $297.0 \mathrm{~m}$ (depth coefficient 0.55). In the testing process, the base station is designed in the section, and the apparent resistivity response characteristics of rock mass under the influence of coal seam stoping schedule are monitored.

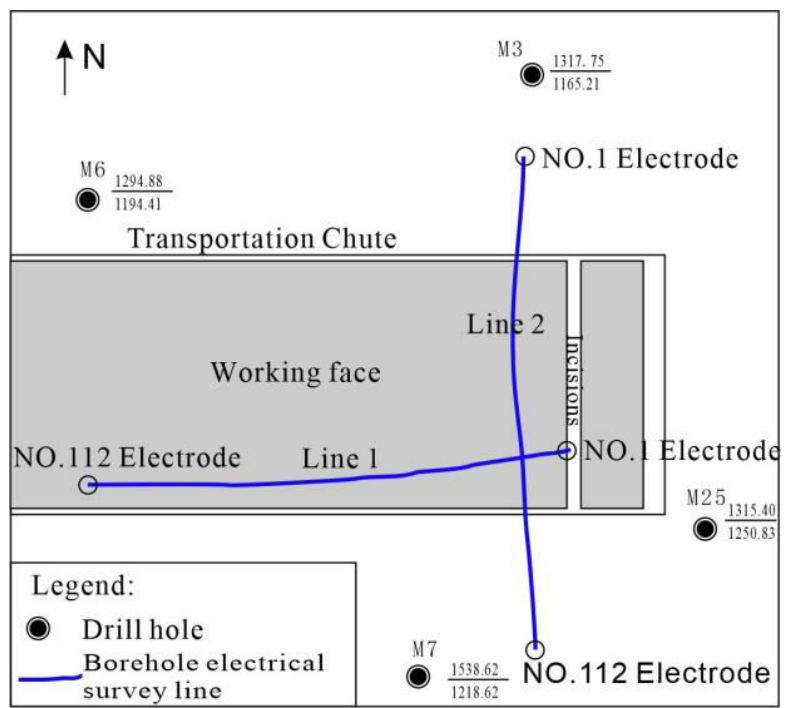

Fig 7: Relative position relationship between working face and survey line 
Fig. 8 is the apparent resistivity profile of No. 1 survey line during the monitoring period. The distance marked on each drawing is the advancing length of the working face. When the coal seam is not mined, the background values are distributed in the range of $0-450 \Omega \cdot \mathrm{m}$ (Fig. 8a). In most areas of the upper loess strata, the apparent resistivity ranges from $220-450 \Omega \cdot \mathrm{m}$. In the Jurassic strata, the resistivity is generally lower than $220 \Omega \cdot \mathrm{m}$, which reflects the difference in electrical parameters of different strata on the whole. The difference in resistivity in individual strata may be caused by the development of rock fractures and uneven water content. When the working face is mined to $210.2 \mathrm{~m}$ back length, the apparent resistivity value of the coal seam roof changes under the influence of mining, forming the mining-influenced area (Fig. 8b). The mining-affected area is mainly formed by the strata above the mined-out area, and the sandstone layer on the roof of the coal seam is rich in water content. The apparent resistivity of the mining-affected area is low due to the influx of sandstone water into mining-induced fractures. When the working face is stope to $300.5 \mathrm{~m}$, the scope of the mining affected area expands (Fig. $8 \mathrm{c}$ ). The apparent resistivity of the strata above the goaf increases because the water in the crack of the roof of the coal seam flows to the goaf. When the working face reaches $404.5 \mathrm{~m}$ (Fig. 8d), the mining affected area above the goaf is further expanded, and the roof of the working face is characterized by high apparent resistivity, which is a caving zone, reflecting the further compaction of the strata in the goaf. At this point, the caving zone and the water-conducting fracture zone have been formed in the profile of the survey line, and the maximum height of the water-conducting fracture zone is $122 \mathrm{~m}$ away from 5 coal. In the monitoring section, consistent with the direction of coal seam mining, the deformation and failure of the rock and soil layer is gradual, and the failure area increases with the increase in mining distance.

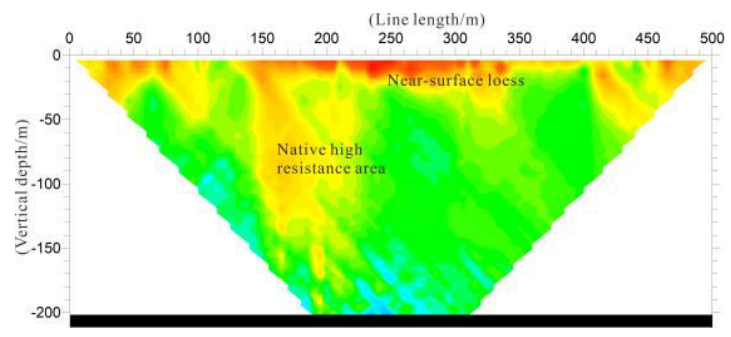

(a) $0 \mathrm{~m}$

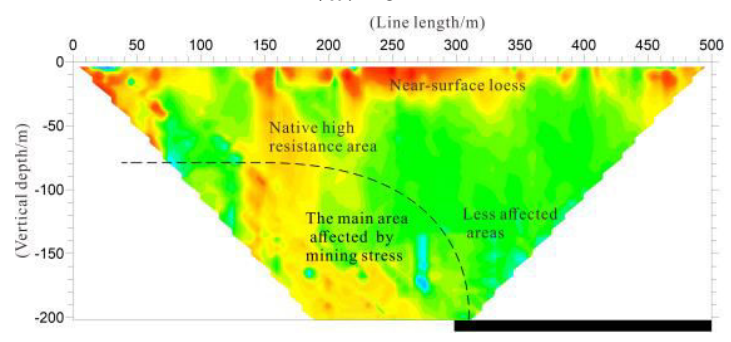

(d) $300.5 \mathrm{~m}$

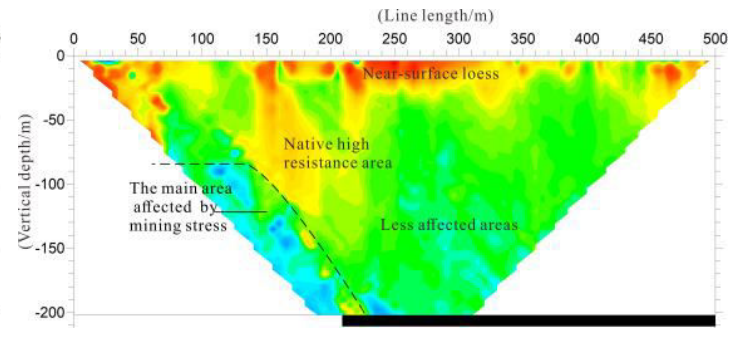

(b) $210.2 \mathrm{~m}$

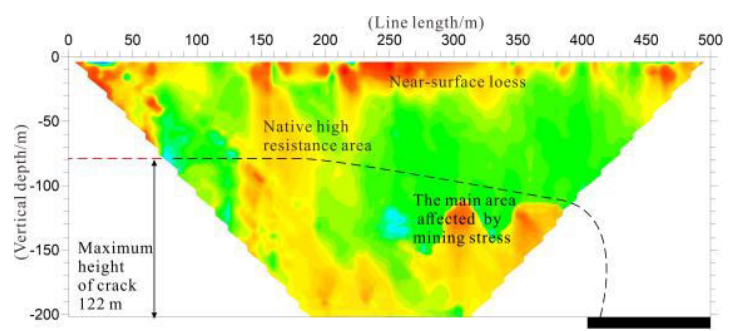

(e) $404.5 \mathrm{~m}$

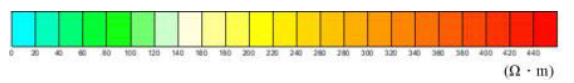

(f) Color code

Fig 8: Response characteristics of apparent resistivity profile of line 1

Fig. 9 shows the apparent resistivity profile of No.2 survey line during the monitoring period. The distance marked on each drawing is the advancing length of the working face. When the coal seam is not mined, the background values are distributed in the range of $0-450 \Omega \cdot \mathrm{m}$ (Fig. 9a). The horizontal distance between the profile of the survey line and the open-off cut is $40 \mathrm{~m}$, which can 
be divided into a high apparent resistivity area near the surface of loess, a medium apparent resistivity area of Jurassic strata, and a low apparent resistivity area of the roof of the water-bearing coal seam. When the stoping face is $210.2 \mathrm{~m}$ away from the open-off cut, the roof of the coal seam is compressed to form the mining-influenced area. Deformation and fracture of rock strata form fracture zones, which are characterized by high values of apparent resistivity (Fig. 9b). Some of the fracture zones are flooded with sandstone water to form low values of apparent resistivity. With the progress of mining, the stoping surface was $210.2 \mathrm{~m}$ away from the open cut. The monitoring profile is completely located above the goaf, and at the same time, the apparent resistivity of the mining-affected area changes (Fig. 9c). On the right side of the profile, the apparent resistivity is high, which is characteristic of mining failure zones. The high apparent resistivity in the middle section is large and continuous. It is speculated that due to the bending and subsidence of the rock strata, the bed separation space is formed in a certain area, and the rock fissures in this area flow to the goaf. The variation in apparent resistivity on the left side of the profile is less affected by mining. When the stoping face is $404.5 \mathrm{~m}$ away from the open-off cut, because the stoping face is far away from the monitoring profile, mining has little influence on the roof structure above the goaf, and its apparent resistivity value is stable in the region (Fig. 9d). At this point, the caving zone and the water-conducting fracture zone are basically formed within the detection range of the profile of the survey line, and the maximum height of the water-conducting fracture zone is $119 \mathrm{~m}$ away from 5 coal roof. Due to the fact that the strata are heterogeneous, the deformation and failure of the same vertical height are different, and the deformation and failure of the strata are not symmetrical.

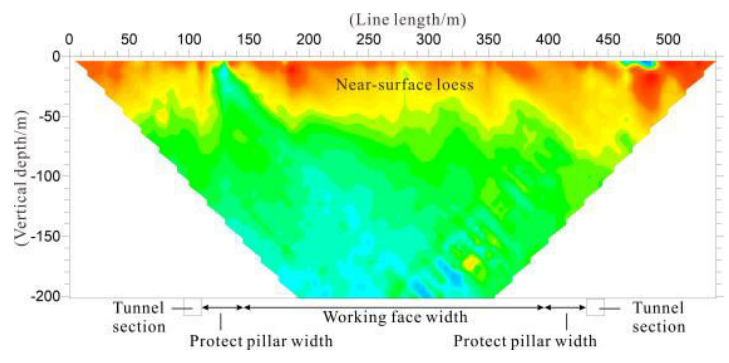

(a) $0 \mathrm{~m}$

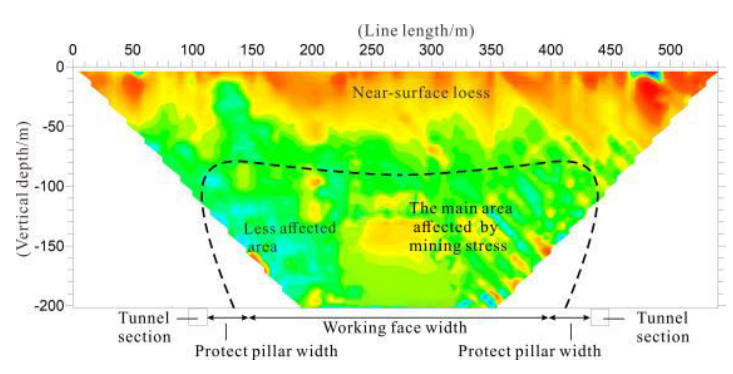

(d) $300.5 \mathrm{~m}$

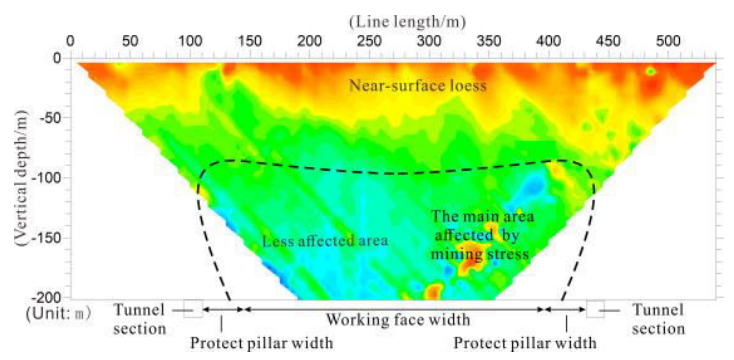

(b) $210.2 \mathrm{~m}$

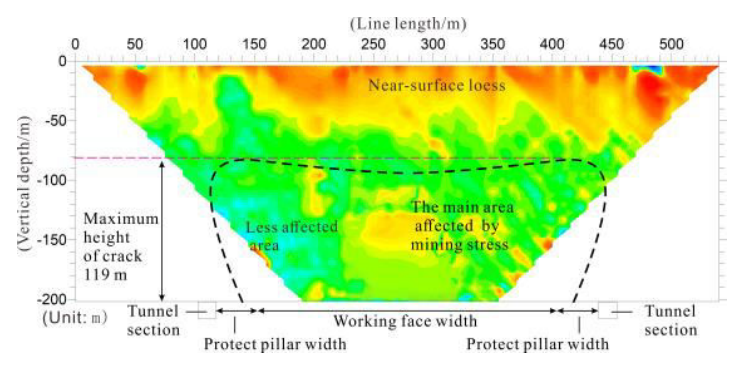

(e) $404.5 \mathrm{~m}$

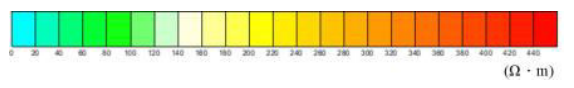

(f) Color code

Fig 9: Apparent resistivity evolution profile of survey line 2

Within the monitoring range, $122 \mathrm{~m}$ above the roof of 5 coal seam is the maximum height of the water-conducting fracture zone in the roof of coal seam. The overall deformation and failure law is obvious, and the stress response characteristics of different layers of overburden are different, 
which leads to the difference in failure characteristics.

Coal mining causes the overlying strata to collapse and bend, thus leading to ground fractures (Fig. 10). This geological dynamic phenomenon can easily cause soil erosion, land subsidence, and other problems. Mining-induced earth fractures can be divided into three types from the perspective of mechanical origin: tension type, compression type, and torsion type. From the distribution morphology, it can be divided into dynamic type and marginal type (Xie et al. 1988). Based on measured data, $\mathrm{Hu}$ et al. (2014) established a mathematical model of the dynamic fracture development cycle $(\mathrm{T})$ and mining process coupling: $\mathrm{T}=2 \mathrm{H}_{0}(1 / \tan \delta+1 / \tan \phi) / \mathrm{V}$. The process of "cracking - closing" of the dynamic ground fissure is analyzed quantitatively. Through observation and analysis, it is found that the mining-induced ground fissure above the edge of the working face exists for a long time, and it is a marginal ground fissure.

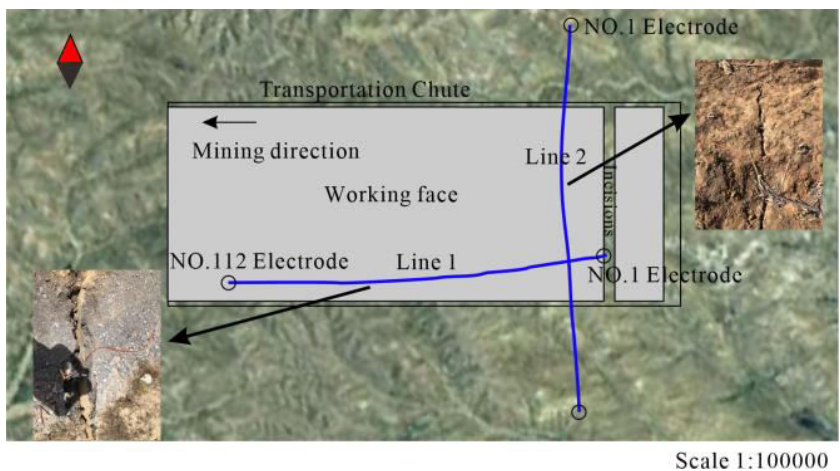

Fig10: Mining induced surface cracks

\section{Conclusions}

In this paper, through theoretical analysis, the microscopic analytical formula of the single-mode parallel electric method influenced by electrode induced polarization effect is obtained. The dual-mode parallel electric method is applied to the detection of roof deformation and failure. On the basis of numerical simulation and field measurements, the following conclusions are drawn.

(1) The effect of single electrode induced polarization on apparent resistivity detection is clarified. The analytical expression of the effect of electrode induced polarization on the apparent resistivity measurement is given.

(2) Based on borehole and logging data, the strata of a coal mine and a working face in Ordos Basin are classified. Based on rock mechanics experiments, a fluid-structure coupling numerical model is constructed to simulate the mining process, and the height of the water-conducting fracture zone is deduced to be $122 \mathrm{~m}$. According to the simulation results of the monitoring points, the vertical stress values above the goaf and the coal seam strike have zoning characteristics.

(3) According to the electrical response law of the rock and soil layer, the height of the water-conducting fracture zone is $122 \mathrm{~m}$. These results are consistent with the numerical simulation results and can be mutually verified. The average coal recovery thickness is $11 \mathrm{~m}$, and the maximum fracture height/average mining thickness ratio is 11.1 These results could be leveraged in future research on the deformation and failure of the roof near the working face.

(4) The surface has a thick loose layer, and a long-term marginal ground fissure appears under the mining operation, which brings with it risks in terms of soil erosion and land subsidence. Therefore, attention should be paid to surface restoration measures. 


\section{Data Availability}

The data used to support the findings of this study are included within the article.

\section{Conflicts of Interest}

The authors declare that there are no conflicts of interest.

Acknowledgements The study was supported by the National Natural Science Foundation of China (Grant No. 41877268, and Grant No. 42074148) and the Graduate Innovation Fund Project of the Anhui University of Science and Technology (Grant No. 2020CX2003 and 2019CX1002). We are also grateful to Ou Yuanchao and Tian Yutong for their assistance in field testing and data processing.

\section{References}

Fu Yuping, Song Guobao, Xing Pingwei (2012) Simulation study on fracture evolution law of roof strata in shallow and thick coal seam with large mining height. J China Coal Soc 37(03): 366-371.

Chen Chao, Hu Zhenqi (2018) Research progress on formation mechanism of mining-induced ground fractures in China. J China Coal Soc 43(03): 810-823.

Yu Z, Zhu S, Wu Y (2019) Study on the structural characteristics of the overburden under thick loose layer and thin-bed rock for safety of mining coal seam, ENVIRON EARTH SCI 79(1).

Qian Minggao, Shi Pingwu (2003) Mining Pressure and Strata Control. China U Min Techno Press 45-50

Wang Jingming (2000) The Theory and Application of Ground Fissures and Their Disasters. Shanxi Sci Techno Press 75-81

Li Jianwei, Liu Changyou, Bu Qingwei (2020) Spatio-temporal evolution of overburden fissures in shallow thick coal seam mining. J Min Safety Eng 37(02): 238-246

Xu Jialin, Qian Minggao, Zhu Weibing (2005) Research on the influence of overburden key layer on surface subsidence. Rock Mech Eng 05: 787-791

Guo Wenbing, Zhao Gaobo, Lou Gaozhong (2019) A new method of predicting the height of the fractured water-conducting zone due to high-intensity longwall coal mining in China. Rock Mech Rock Eng 52(08): 2789-2802

Huang Wanpeng, Gao Yanfa, Wang Bo (2017) Evolution rule and development height of permeable fractured zone under combined-strata structure. J Min Safety Eng 34(02): 330-335

An Tailong, Wang Lianguo, Pu Hai (2010) Numerical simulation study on development law of water-conducting fracture zone in shallow coal seam. Mining Research and Development 30(01): 33-36

Liu Hui, Liu Xiaoyang, Deng Kazhong (2016) Developing law of sliding ground fissures based on numerical simulation using UDEC". J China Coal Soc 41(03): 625-632

Liu Hui, Deng Kazhong (2014) Development Law and Control Technology of Mining Ground Fissures in Western Loess Gully Region. China University of Mining and Technology Press $105-108$

Chai Jing, Yang Yuyu, Ouyang Yibo (2021) A Comparative Study of Optical Measurement Methods for Deformation and Failure Simulation Test of Overburden in Stope. J China Coal Soc 46(01): 154-163

Dan Z, Wang J. C, Zhang P S (2017) Internal strain monitoring for coal mining similarity model 
based on distributed fiber optical sensing. Measurement 97: 234-241

Sun Q, Meng G, Sun K (2020) Physical simulation experiment on prevention and control of water inrush disaster by backfilling mining under aquifer. ENVIRON EARTH SCI 79(18)

Sun Qingxian, Mou Yi, Yang Xinliang (2013) Comprehensive detection of "Two Zones" height of overlying rock with large mining height in Hongliu Coal Mine. J China Coal Soc 38(02): 283-286

Wu R, Hu Z, Hu X (2020) Principle of using borehole electrode current method to monitor the overburden stratum failure after coal seam mining and its application. J APPL GEOPHYS 179: 104-111

Ou Y. C, Zhang P, Liu C (2020) Comparative study of 3D joint inversion based on multi-section resistivity data. AIP ADV 10(8): 85-102

Liu S, Yang K, Tang C. Rupture and migration law of disturbed overburden during slicing mining of steeply dipping thick coal seam. ADV CIV ENG 04: 1-11

Xia Z. Y, Tan Z Y (2121) Study on instability mechanism of extraction structure under undercut space based on thin plate theory in block caving method. Shock Vib 02: 1-11

Zhou W. L, Zhang P S , Wu R X (2019) Dynamic monitoring study on the characteristics of deformation and failure of extra-thick coal seam floor in deep mining. J Applied Geophys 163: $132-138$

Liu Shengdong, Liu Jing, and Qi Jun (2019) Applied technologies and new advances of parallel electrical method in mining geophysics. J China Coal Soc 44(08): 2336-2345

Yang C, Liu S. D, Feng Y, Yang. H (2018) Influence of electrode polarization on the potential of DC electrical exploration. J China Coal Soc 149: 63-76

Cheng Zhiping (2007) Tutorial of electrical exploration. Metallurgical Industry Press, 125-128

Xie Guanglin (1988) Ground Fissure. Seismological Press 100-105

Hu Zhenqi, Wang Xinjing, He Anmin (2014) Distribution characteristic and development rules of ground fissures due to coal mining in windy and sandy region. J China Coal Soc 39(01): $11-18$ 